\title{
Local well-posedness of the inertial Qian-Sheng's Q-tensor dynamical model near uniaxial equilibrium
}

\author{
Xiaoyuan Wang ${ }^{1}$, Sirui $\mathrm{Li}^{1,2^{*}}$ (D) and Tingting Wang ${ }^{1}$
}

"Correspondence: srli@gzu.edu.cn
'School of Mathematics and
Statistics, Guizhou University,
Guiyang, China
${ }^{2}$ School of Mathematical Sciences,
Zhejiang University, Hangzhou,
China

"Correspondence: srli@gzu.edu.cn School of Mathematics and Guiyang, China Zhejiang University, Hangzhou,

\begin{abstract}
We consider the inertial Qian-Sheng's Q-tensor dynamical model for the nematic liquid crystal flow, which can be viewed as a system coupling the hyperbolic-type equations for the $Q$-tensor parameter with the incompressible Navier-Stokes equations for the fluid velocity. We prove the existence and uniqueness of local in time strong solutions to the system with the initial data near uniaxial equilibrium. The proof is mainly based on the classical Friedrich method to construct approximate solutions and the closed energy estimate.
\end{abstract}

MSC: 35Q35; 35Q30; 76D03; 76D05

Keywords: Liquid crystals; Qian-Sheng's inertial system; Q-tensor; Local strong solutions

\section{Introduction}

Liquid crystals present a state of matter with properties between liquid and solid. The simplest form of liquid crystals is the nematic phase, which exhibits long-range orientational order but no positional order. Generally speaking, there are two primary continuum theories to describe nematic liquid crystal flow: the Ericksen-Lesile theory and the Landaude Gennes theory. In the former one, the local alignment of molecules is described by a unit vector, which completely neglects molecular details. In contrast, the latter gives a more complex description of the local behavior of molecular alignments, such as line defects and biaxial configurations. This theory uses a symmetric and traceless tensor $Q(\mathbf{x})$ to characterize the alignment behavior of molecular orientations. Physically, $Q(\mathbf{x})$ can be defined as the second-order traceless moment of $f$ :

$$
Q(\mathbf{x})=\int_{\mathbb{S}^{2}}\left(\mathbf{m} \mathbf{m}-\frac{1}{3} \mathbf{I}\right) f(\mathbf{x}, \mathbf{m}) \mathrm{d} \mathbf{m},
$$

where $f(\mathbf{x}, \mathbf{m})$ is the density distribution function with the orientation parallel to $\mathbf{m}$ at material point $\mathbf{x}$. The tensor $Q(\mathbf{x})$ is said to be isotropic if all its eigenvalues are zero, uniaxial if it has only two different eigenvalues, and biaxial if its three eigenvalues are different

(c) The Author(s) 2021. This article is licensed under a Creative Commons Attribution 4.0 International License, which permits use, sharing, adaptation, distribution and reproduction in any medium or format, as long as you give appropriate credit to the original author(s) and the source, provide a link to the Creative Commons licence, and indicate if changes were made. The images or other third party material in this article are included in the article's Creative Commons licence, unless indicated otherwise in a credit line to the material. If material is not included in the article's Creative Commons licence and your intended use is not permitted by statutory regulation or exceeds the permitted use, you will need to obtain permission directly from the copyright holder. To view a copy of this licence, visit http://creativecommons.org/licenses/by/4.0/. 
from each other. When $Q(\mathbf{x})$ is uniaxial, it can be written as

$$
Q(\mathbf{x})=S\left(\mathbf{n n}-\frac{1}{3} \mathbf{I}\right), \quad \mathbf{n} \in \mathbb{S}^{2}
$$

where $S \in \mathbb{R}$ is the scalar order parameter. When $Q(\mathbf{x})$ is biaxial, it can be written as

$$
Q(\mathbf{x})=S\left(\mathbf{n n}-\frac{1}{3} \mathbf{I}\right)+R\left(\mathbf{n}^{\prime} \mathbf{n}^{\prime}-\frac{1}{3} \mathbf{I}\right), \quad \mathbf{n}, \mathbf{n}^{\prime} \in \mathbb{S}^{2}, \mathbf{n} \cdot \mathbf{n}^{\prime}=0, S, R \in \mathbb{R}
$$

The Landau-de Gennes free energy functional is given as follows:

$$
\begin{aligned}
\mathcal{F}(Q, \nabla Q)= & \int_{\mathbb{R}^{3}}\left\{-\frac{a}{2} \operatorname{Tr}\left(Q^{2}\right)-\frac{b}{3} \operatorname{Tr}\left(Q^{3}\right)+\frac{c}{4}\left(\operatorname{Tr}\left(Q^{2}\right)\right)^{2}\right. \\
& \left.+\frac{1}{2}\left(L_{1}|\nabla Q|^{2}+L_{2} Q_{i j, j} Q_{i k, k}+L_{3} Q_{i j, k} Q_{i k, j}\right)\right\} \mathrm{d} \mathbf{x} \\
\stackrel{\text { def }}{=} & \int_{\mathbb{R}^{3}}\left(f_{b}(Q)+f_{e}(\nabla Q)\right) \mathrm{d} \mathbf{x},
\end{aligned}
$$

where $a, b, c$ are nonnegative coefficients depending on the material and temperature, and $L_{i}(i=1,2,3)$ are material-dependent elastic coefficients. $f_{b}$ is the bulk energy density describing the isotropic-nematic phase transition, while the elastic energy density $f_{e}$ penalizes spatial non-homogeneities. For detailed introductions one is referred to $[5,13]$.

In the Landau-de Gennes framework, there exist two representative Q-tensor models, directly derived by a variational method, describing the hydrodynamics of nematic liquid crystals: the Beris-Edwards model [3] and the Qian-Sheng model [16]. The two models are, respectively, a system coupling the equation of $Q$-tensor order parameters with the time evolution equation of the fluid velocity. In this paper, we are concerned with the following Qian-Sheng model [16] with the inertial density:

$$
\begin{aligned}
& J \ddot{Q}+\mu_{1} \dot{Q}=\mathbf{H}-\frac{\mu_{2}}{2} \mathbf{D}+\mu_{1}[\boldsymbol{\Omega}, Q], \\
& \frac{\partial \mathbf{v}}{\partial t}+\mathbf{v} \cdot \nabla \mathbf{v}=-\nabla p+\nabla \cdot\left(\sigma+\sigma^{d}\right), \\
& \nabla \cdot \mathbf{v}=0,
\end{aligned}
$$

where $J$ stands for the small inertial coefficient, and the inertial term $\ddot{Q}=\left(\partial_{t}+\mathbf{v} \cdot \nabla\right) \dot{Q}$ is the material derivative of $\dot{Q}=\left(\partial_{t}+\mathbf{v} \cdot \nabla\right) Q$. In addition, the viscous stress $\sigma$, the distortion stress $\sigma^{d}$ and the molecular field $\mathbf{H}$ are, respectively, defined by

$$
\begin{aligned}
\sigma= & \beta_{1} Q(Q: \mathbf{D})+\beta_{4} \mathbf{D}+\beta_{5} \mathbf{D} \cdot Q+\beta_{6} Q \cdot \mathbf{D}+\beta_{7}\left(\mathbf{D} \cdot Q^{2}+Q^{2} \cdot \mathbf{D}\right) \\
& +\frac{\mu_{2}}{2}(\dot{Q}-[\mathbf{\Omega}, Q])+\mu_{1}[Q,(\dot{Q}-[\mathbf{\Omega}, Q])], \\
\sigma_{i j}^{d}= & -\frac{\partial \mathcal{F}}{\partial Q_{k l, j}} \partial_{i} Q_{k l}, \\
\mathbf{H}_{i j}= & -\left(\frac{\delta \mathcal{F}(Q, \nabla Q)}{\delta Q}\right)_{i j}=-\frac{\partial \mathcal{F}}{\partial Q_{i j}}+\partial_{k}\left(\frac{\partial \mathcal{F}}{\partial Q_{i j, k}}\right) \stackrel{\text { def }}{=}-\mathcal{T}(Q)-\mathcal{L}(Q),
\end{aligned}
$$


where the two operators $\mathcal{T}$ and $\mathcal{L}$ are, respectively, given by

$$
\begin{aligned}
& \mathcal{T}(Q)=-a Q-b Q^{2}+c|Q|^{2} Q+\frac{1}{3} b|Q|^{2} \mathbf{I}, \\
& (\mathcal{L}(Q))_{k l}=-\left(L_{1} \Delta Q_{k l}+\frac{1}{2}\left(L_{2}+L_{3}\right)\left(Q_{k m, m l}+Q_{l m, m k}-\frac{2}{3} \delta_{k l} Q_{i j, i j}\right)\right) .
\end{aligned}
$$

The constants $\beta_{1}, \beta_{4}, \beta_{5}, \beta_{6}, \beta_{7}, \mu_{1}$, and $\mu_{2}$ in (1.5) are viscosity coefficients. The coefficients satisfy the following relation:

$$
\beta_{6}-\beta_{5}=\mu_{2}
$$

It is worth emphasizing that, to be compared with the original Qian-Sheng model in [16], a new viscosity term $\beta_{7}\left(D_{i k} Q_{k l} Q_{l j}+Q_{i k} Q_{k l} D_{l j}\right)$ in (1.5) is added to ensure that the energy of the system will always dissipate without assuming any relation between $\beta_{5}$ and $\beta_{6}$. The detailed discussion of the dissipative relation can be found in recent work [9].

For the Q-tensor dynamical model of liquid crystals, there has been published much analytical work. We only recall some relevant results here. Concerning the Beris-Edwards system, the well-posedness results on whole space and bounded domain can be found in $[8,14,15]$ and $[1,2,11]$, respectively. For the inertial Qian-Sheng model, De Anna and Zarnescu [4] investigated the local well-posdedness for bounded initial data and global well-posedness under the assumptions of the small initial data. For the non-viscous version of the Qian-Sheng model, Feireisl et al. [6] proved global existence of the dissipative solution which is inspired by that of the incompressible Euler equations. There is some interesting work, devoted to exploring the relation between different dynamical theories for liquid crystals. For example, by the Hilbert expansion method, Wang-Zhang-Zhang [19] rigorously justified that the strong solution to the non-inertial Beris-Edwards model converges to the solution to the Ericksen-Leslie model. In the same spirit, Li-Wang [9] extended this work, and rigorously proved the connection between the inertial Qian-Sheng model and the full inertial Ericksen-Leslie model. A unified formulation for liquid crystal modeling was put forward by Han et al. in [7] to establish relations between microscopic theories and macroscopic theories.

In [4], the well-posedness results rely on the assumption that the solution decays fast enough at infinity. However, during the physical modeling process, the liquid crystal system is not generally isotropic but certain nonzero uniaxial or biaxial equilibrium at infinity. Therefore, the main goal of this paper is to study the local well-posedness of the strong solution for the inertial Qian-Sheng system with the initial data near uniaxial equilibrium.

The rest of this paper is organized as follows. In Sect. 2, we state the notational conventions and some technical lemmas, and then present the main result of this paper. In Sect. 3, based on the classical Friedrich method and the closed energy estimate, we prove the local well-posedness of the inertial Qian-Sheng's Q-tensor dynamical model, when the solution to the system tends to the uniaxial equilibrium state at infinity. 


\section{Preliminaries and the main result}

\subsection{Notations and convections}

The Einstein summation convention is used in this paper. The configuration space of the $Q$-tensor is the set of symmetric, traceless $3 \times 3$-matrices, that is,

$$
\mathbb{S}_{0}^{3} \stackrel{\text { def }}{=}\left\{Q \in \mathbb{R}^{3 \times 3}: Q_{i j}=Q_{j i}, Q_{i i}=0\right\}
$$

which is endowed with the inner product $Q_{1}: Q_{2}=Q_{1 i j} Q_{2 i j}$. The Frobenius norm on $\mathbb{S}_{0}^{3}$ is defined as $|Q| \stackrel{\text { def }}{=} \sqrt{\operatorname{Tr} Q^{2}}=\sqrt{Q_{i j} Q_{i j}}$. For two tensors $A, B \in \mathbb{S}_{0}^{3}$ we denote $(A \cdot B)_{i j}=A_{i k} B_{k j}$ and $A: B=A_{i j} B_{i j}$, and $[A, B]=A \cdot B-B \cdot A$. For any $Q_{1}, Q_{2} \in L^{2}\left(\mathbb{R}^{3}\right)^{3 \times 3}$, the corresponding inner product is defined as

$$
\left\langle Q_{1}, Q_{2}\right\rangle \stackrel{\text { def }}{=} \int_{\mathbb{R}^{3}} Q_{1 i j}(\mathbf{x}): Q_{2 i j}(\mathbf{x}) \mathrm{d} \mathbf{x} .
$$

We denote by $\mathbf{n}_{1} \otimes \mathbf{n}_{2}$ the tensor product of two vectors $\mathbf{n}_{1}$ and $\mathbf{n}_{2}$, and omit the symbol $\otimes$ for simplicity. We use $f_{, i}$ to denote $\partial_{i} f$ and $\mathbf{I}$ to denote the $3 \times 3$ identity tensor. In addition, the superscripted dot denotes the material derivative, i.e., $\dot{f}=\left(\partial_{t}+\mathbf{v} \cdot \nabla\right) f$, where the fluid velocity $\mathbf{v}$ can be understood from the context. We also define the commutator $\llbracket \nabla^{s}, f \rrbracket g=\nabla^{s}(f g)-f \nabla^{s} g$.

\subsection{Useful lemmas}

The following product estimates and commutator estimates are well-known, see $[10,17]$ for example, and they are frequently used in this paper.

Lemma 2.1 Let $s \geq 0$. Then, for any multi-index $\alpha, \beta$,

$$
\begin{aligned}
& \left\|\partial^{\alpha} f \partial^{\beta} g\right\|_{H^{s}} \leq C\left(\|f\|_{L^{\infty}}\|g\|_{H^{s+|\alpha|+|\beta|}}+\|g\|_{L^{\infty}}\|f\|_{H^{s+|\alpha|+|\beta|}}\right) \\
& \left\|\partial^{\alpha} f \partial^{\beta} g\right\|_{H^{s}} \leq C\|f\|_{H^{s+|\alpha|+|\beta|}}\|g\|_{H^{s+|\alpha|+|\beta|}}, \quad \text { if } s+|\alpha|+|\beta| \geq 2 .
\end{aligned}
$$

In particular, we have

$$
\begin{aligned}
& \|f g\|_{H^{s}} \leq C\left(\|f\|_{L^{\infty}}\|g\|_{H^{s}}+\|g\|_{L^{\infty}}\|f\|_{H^{s}}\right) ; \\
& \|f g\|_{H^{s}} \leq C\|f\|_{H^{s}}\|g\|_{H^{s}}, \quad \text { if } s \geq 2 ; \\
& \|f g\|_{H^{k}} \leq C \min \left\{\|f\|_{H^{k}}\|g\|_{H^{2}},\|f\|_{H^{2}}\|g\|_{H^{k}}\right\}, \quad \text { if } 0 \leq k \leq 2 .
\end{aligned}
$$

Lemma 2.2 Let a be a multiple index. We have

$$
\left\|\llbracket \partial^{a}, g \rrbracket f\right\|_{L^{2}} \leq C\left(\|\nabla g\|_{L^{\infty}}\|f\|_{H^{|a|-1}}+\|\nabla g\|_{H^{|a|-1}}\|f\|_{L^{\infty}}\right) .
$$

In particular, if $|a| \geq 2$, we have

$$
\begin{aligned}
& \left\|\llbracket \partial^{a}, g \rrbracket f\right\|_{L^{2}} \leq C\|g\|_{H^{|a|+1}}\|f\|_{H^{|a|-1}}, \\
& \left\|\llbracket \partial^{a+1}, g \rrbracket f\right\|_{L^{2}} \leq C\|g\|_{H^{|a|+1}}\|f\|_{H^{|a|-1}} .
\end{aligned}
$$


The following energy dissipation relation can be found in [9].

Lemma 2.3 Assume that $\beta_{1}, \beta_{4}, \mu_{1}>0, \beta_{7} \geq 0$, and $\beta_{4}-\frac{\mu_{2}^{2}}{4 \mu_{1}}>0$. Then, for any smooth solution $(\mathbf{v}, Q)$ of the inertial Qian-Sheng system (1.2)-(1.4),

$$
\begin{aligned}
\frac{\mathrm{d}}{\mathrm{d} t}( & \left.\int_{\mathbb{R}^{3}} \frac{1}{2}\left(|\mathbf{v}|^{2}+J|\dot{Q}|^{2}\right) \mathrm{d} \mathbf{x}+\mathcal{F}(Q, \nabla Q)\right) \\
= & -\beta_{1}\|Q: \mathbf{D}\|_{L^{2}}^{2}-\left(\beta_{4}-\frac{\mu_{2}^{2}}{4 \mu_{1}}\right)\|\mathbf{D}\|_{L^{2}}^{2}-\left(\beta_{5}+\beta_{6}\right)\langle\mathbf{D} \cdot Q, \mathbf{D}\rangle \\
& -2 \beta_{7}\|\mathbf{D} \cdot Q\|_{L^{2}}^{2}-\mu_{1}\left\|\dot{Q}-[\boldsymbol{\Omega}, Q]+\frac{\mu_{2}}{2 \mu_{1}} \mathbf{D}\right\|_{L^{2}}^{2} .
\end{aligned}
$$

Moreover, if one of the following assumptions holds: (i) $\beta_{5}+\beta_{6}=0$ if $\beta_{7}=0$, (ii) $\left(\beta_{5}+\beta_{6}\right)^{2}<$ $8 \beta_{7}\left(\beta_{4}-\frac{\mu_{2}^{2}}{4 \mu_{1}}\right)$ if $\beta_{7} \neq 0$, then the right hand side in (2.1) is non-positive.

We give some results about critical points. A tensor $Q_{0}$ is called a critical point of $f_{b}(Q)$ if $\mathcal{T}\left(Q_{0}\right):=\left.\frac{\partial f_{b}}{\partial Q}\right|_{Q=Q_{0}}=0$. The following characterization of critical points can be obtained from $[12,19]$.

Lemma 2.4 $\mathcal{T}(Q)=0$ if and only if $Q=S\left(\mathbf{n n}-\frac{1}{3} \mathbf{I}\right)$ for some $\mathbf{n} \in \mathbb{S}^{2}$, where $S=0$ or a solution of $2 c S^{2}-b S+3 a=0$, that is,

$$
S_{1}=\frac{b+\sqrt{b^{2}+24 a c}}{4 c} \text { or } \quad S_{2}=\frac{b-\sqrt{b^{2}+24 a c}}{4 c} .
$$

Moreover, the critical point $Q_{0}=S\left(\mathbf{n n}-\frac{1}{3} \mathbf{I}\right)$ is stable if $S=S_{1}$.

Given a critical point $Q_{0}=S\left(\mathbf{n n}-\frac{1}{3} \mathbf{I}\right)$, the linearized operator $\mathcal{H}_{Q_{0}}$ of $\mathcal{T}(Q)$ around $Q_{0}$ is given by

$$
\mathcal{H}_{Q_{0}}(Q)=a Q-b\left(Q_{0} \cdot Q+Q \cdot Q_{0}\right)+c\left|Q_{0}\right|^{2} Q+2\left(Q_{0}: Q\right)\left(c Q_{0}+\frac{b}{3} \mathbf{I}\right) .
$$

\subsection{Main results}

Throughout this paper, we assume that the viscosity coefficients satisfy $\beta_{1}, \beta_{4}, \mu_{1}>0, \beta_{7} \geq$ 0 , and $\beta_{4}-\frac{\mu_{2}^{2}}{4 \mu_{1}}>0$, and the elastic coefficients $L_{i}(i=1,2,3)$ satisfy $L_{1}>0, L_{1}+L_{2}+L_{3}>0$, and the inertial coefficient $J$ is positive, and $J \ll \mu_{1}$.

The main assertion of this paper is stated as follows.

Theorem 2.1 Let $s \geq 2$ be an integer, $\mathbf{n}^{*} \in \mathbb{S}^{2}$ is a constant vector and $Q^{*}=S\left(\mathbf{n}^{*} \mathbf{n}^{*}-\frac{1}{3} \mathbf{I}\right)$. If the initial data fulfills

$$
\mathbf{v}_{I}(\mathbf{x}) \in H^{s}\left(\mathbb{R}^{3}\right), \quad Q_{I}(\mathbf{x})-Q^{*} \in H^{s+1}\left(\mathbb{R}^{3}\right), \quad \dot{Q}_{I}(\mathbf{x}) \in H^{s}\left(\mathbb{R}^{3}\right),
$$

for all $\mathbf{x} \in \mathbb{R}^{3}$, then there exist $T>0$ and a unique solution $(\mathbf{v}, Q)$ of the inertial Qian-Sheng Q-tensor system (1.2)-(1.4) on $[0, T]$, such that $\mathbf{v}(0, \mathbf{x})=\mathbf{v}_{I}(\mathbf{x}), Q(0, \mathbf{x})=Q_{I}(\mathbf{x})$, and

$$
\begin{aligned}
& \mathbf{v} \in L^{\infty}\left([0, T] ; H^{s}\left(\mathbb{R}^{3}\right)\right) \cap L^{2}\left([0, T] ; H^{s+1}\left(\mathbb{R}^{3}\right)\right), \\
& Q-Q^{*} \in L^{\infty}\left([0, T] ; H^{s+1}\left(\mathbb{R}^{3}\right)\right), \quad \dot{Q} \in L^{\infty}\left([0, T] ; H^{s}\left(\mathbb{R}^{3}\right)\right) .
\end{aligned}
$$




\section{Local well-posedness for the inertial Qian-Sheng model}

This section is devoted to the proof of the local well-posedness result for the inertial QianSheng model with the initial data near uniaxial equilibrium. The main framework of our proof follows the strategy in [18]. We divide the proof of Theorem 2.1 into four steps.

Step 1. Construction of approximate solutions. Based on the classical Friedrich method, we construct the approximate system of the inertial Qian-Sheng model (1.2)-(1.4). We define the mollification operator

$$
\mathcal{J}_{\varepsilon} f(\xi) \stackrel{\text { def }}{=} \mathrm{F}^{-1}\left(\mathbf{1}_{|\xi| \leq \frac{1}{\varepsilon}} \mathrm{F} f\right)
$$

where $\mathrm{F}$ is the Fourier transform. Assume that $\mathbb{P}$ is the Leray projection operator from a vector field into the corresponding divergence-free field.

Then the approximate system associated with (1.2)-(1.4) is given by

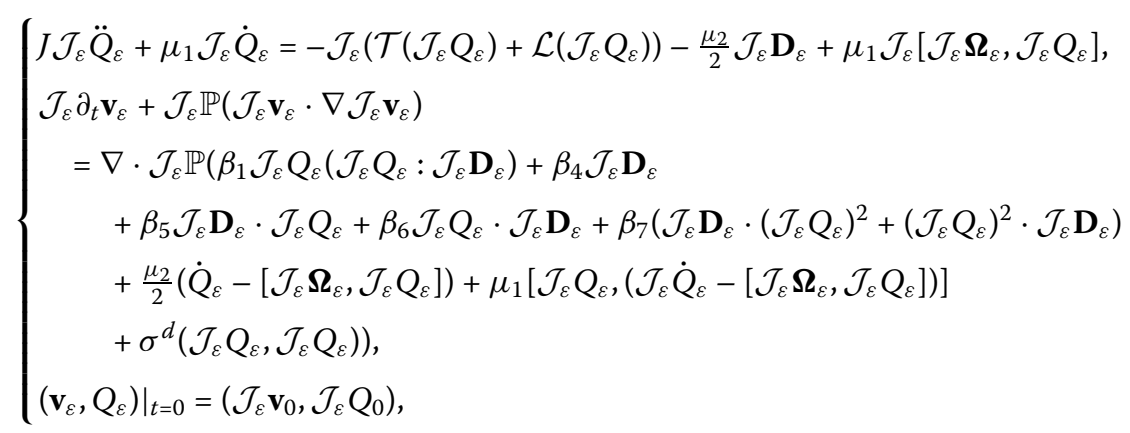

where the material derivative $\dot{Q}_{\varepsilon} \stackrel{\text { def }}{=} \partial_{t} Q_{\varepsilon}+\mathcal{J}_{\varepsilon}\left(\mathcal{J}_{\varepsilon} \mathbf{v}_{\varepsilon} \cdot \nabla \mathcal{J}_{\varepsilon} Q_{\varepsilon}\right)$, and $\mathcal{T}\left(\mathcal{J}_{\varepsilon} Q_{\varepsilon}\right)$ and $\mathcal{L}\left(\mathcal{J}_{\varepsilon} Q_{\varepsilon}\right)$ are, respectively, defined as

$$
\begin{aligned}
\mathcal{T}\left(\mathcal{J}_{\varepsilon} Q_{\varepsilon}\right)= & -a \mathcal{J}_{\varepsilon} Q_{\varepsilon}-b\left(\mathcal{J}_{\varepsilon} Q_{\varepsilon}\right)^{2}+c\left|\mathcal{J}_{\varepsilon} Q_{\varepsilon}\right|^{2} \mathcal{J}_{\varepsilon} Q_{\varepsilon}+\frac{1}{3} b\left|\mathcal{J}_{\varepsilon} Q_{\varepsilon}\right|^{2} \mathbf{I} \\
\mathcal{L}\left(\mathcal{J}_{\varepsilon} Q_{\varepsilon}\right)_{k l}= & -\left(L_{1} \Delta \mathcal{J}_{\varepsilon}\left(Q_{\varepsilon}\right)_{k l}+\frac{1}{2}\left(L_{2}+L_{3}\right)\left(\mathcal{J}_{\varepsilon}\left(Q_{\varepsilon}\right)_{k m, m l}+\mathcal{J}_{\varepsilon}\left(Q_{\varepsilon}\right)_{l m, m k}\right.\right. \\
& \left.\left.-\frac{2}{3} \delta_{k l} \mathcal{J}_{\varepsilon}\left(Q_{\varepsilon}\right)_{i j, i j}\right)\right)
\end{aligned}
$$

The above system can be regarded as an ODE system in $L^{2}\left(\mathbb{R}^{3}\right)$. Then, applying the Cauchy-Lipshitz theorem, there exist a strictly maximal time $T_{\varepsilon}$ and a unique solution $\left(\mathbf{v}_{\varepsilon}, Q_{\varepsilon}\right)$, which is continuous in time with a value in $H^{k}\left(\mathbb{R}^{3}\right)$ for any $k \geq 0$. Since $\mathcal{J}_{\varepsilon}^{2}=\mathcal{J}_{\varepsilon}$ and $\mathbb{P}$ is a self-adjoint operator in $L^{2}\left(\mathbb{R}^{3}\right)$, the pair $\left(\mathcal{J}_{\varepsilon} \mathbf{v}_{\varepsilon}, \mathcal{J}_{\varepsilon} Q_{\varepsilon}\right)$ is also a solution of the previous system. Therefore, the uniqueness of the solution leads to $\left(\mathcal{J}_{\varepsilon} \mathbf{v}_{\varepsilon}, \mathcal{J}_{\varepsilon} Q_{\varepsilon}\right)=\left(\mathbf{v}_{\varepsilon}, Q_{\varepsilon}\right)$, and thus $\left(\mathbf{v}_{\varepsilon}, Q_{\varepsilon}\right)$ satisfies the following system:

$$
\left\{\begin{aligned}
J \ddot{Q}_{\varepsilon} & +\mu_{1} \dot{Q}_{\varepsilon}=-\mathcal{J}_{\varepsilon}\left(\mathcal{T}\left(Q_{\varepsilon}\right)+\mathcal{L}\left(Q_{\varepsilon}\right)\right)-\frac{\mu_{2}}{2} \mathbf{D}_{\varepsilon}+\mu_{1} \mathcal{J}_{\varepsilon}\left[\boldsymbol{\Omega}_{\varepsilon}, Q_{\varepsilon}\right], \\
\partial_{t} \mathbf{v}_{\varepsilon} & +\mathcal{J}_{\varepsilon} \mathbb{P}\left(\mathbf{v}_{\varepsilon} \cdot \nabla \mathbf{v}_{\varepsilon}\right) \\
= & \nabla \cdot \mathcal{J}_{\varepsilon} \mathbb{P}\left(\beta_{1} Q_{\varepsilon}\left(Q_{\varepsilon}: \mathbf{D}_{\varepsilon}\right)+\beta_{4} \mathbf{D}_{\varepsilon}\right. \\
& +\beta_{5} \mathbf{D}_{\varepsilon} \cdot Q_{\varepsilon}+\beta_{6} Q_{\varepsilon} \cdot \mathbf{D}_{\varepsilon}+\beta_{7}\left(\mathbf{D}_{\varepsilon} \cdot Q_{\varepsilon}^{2}+Q_{\varepsilon}^{2} \cdot \mathbf{D}_{\varepsilon}\right) \\
& \left.\quad+\frac{\mu_{2}}{2}\left(\dot{Q}_{\varepsilon}-\left[\boldsymbol{\Omega}_{\varepsilon}, Q_{\varepsilon}\right]\right)+\mu_{1}\left[Q_{\varepsilon},\left(\dot{Q}_{\varepsilon}-\left[\boldsymbol{\Omega}_{\varepsilon}, Q_{\varepsilon}\right]\right)\right]+\sigma^{d}\left(Q_{\varepsilon}, Q_{\varepsilon}\right)\right), \\
\left(\mathbf{v}_{\varepsilon},\right. & \left.Q_{\varepsilon}\right)\left.\right|_{t=0}=\left(\mathcal{J}_{\varepsilon} \mathbf{v}_{0}, \mathcal{J}_{\varepsilon} Q_{0}\right) .
\end{aligned}\right.
$$


Step 2. Uniform energy estimates. We define the energy functional $\mathcal{E}(t)$ by

$$
\begin{aligned}
\mathcal{E}(t) \stackrel{\text { def }}{=} & \int_{\mathbb{R}^{3}}\left(|\mathbf{v}|^{2}+\frac{J}{2}\left(\left|\dot{Q}+Q-Q^{*}\right|^{2}+|\dot{Q}|^{2}\right)+\frac{1}{2}\left(\mu_{1}-J\right)\left|Q-Q^{*}\right|^{2}\right. \\
& \left.+\mathcal{L}(Q):\left(Q-Q^{*}\right)+\frac{1}{2}\left|\nabla^{s} \mathbf{v}\right|^{2}+\frac{J}{2}\left|\nabla^{s} \dot{Q}\right|^{2}+\mathcal{L}\left(\nabla^{s} Q\right): \nabla^{s} Q\right) \mathrm{d} \mathbf{x} .
\end{aligned}
$$

Recalling the fact that there exists a constant $L_{0}=\min \left\{L_{1}, L_{1}+L_{2}+L_{3}\right\}>0$ such that (see [19, Lemma 2.5])

$$
\langle\mathcal{L}(Q), Q\rangle=\int_{\mathbb{R}^{3}} f_{e}(\nabla Q) \mathrm{d} \mathbf{x} \geq L_{0}\|\nabla Q\|_{L^{2}}^{2} .
$$

By a Sobolev interpolation, we have

$$
\mathcal{E}(t) \sim\left\|Q-Q^{*}\right\|_{L^{2}}^{2}+\|\nabla Q\|_{H^{s}}^{2}+\|\mathbf{v}\|_{H^{s}}^{2}+\|\dot{Q}\|_{H^{s}}^{2}
$$

Let $\widetilde{Q}_{\varepsilon}=Q_{\varepsilon}-Q^{*}$, then from the expression of $\mathcal{T}(Q)$ we have

$$
\mathcal{T}\left(\widetilde{Q}_{\varepsilon}+Q^{*}\right)=\mathcal{T}\left(Q^{*}\right)+\mathcal{H}_{Q^{*}}\left(\widetilde{Q}_{\varepsilon}\right)+\mathcal{P}_{3}\left(\widetilde{Q}_{\varepsilon}\right)
$$

where $\mathcal{H}_{Q^{*}}$ and $\mathcal{P}_{3}$ are, respectively, defined as

$$
\begin{aligned}
& \mathcal{H}_{Q^{*}}(Q) \stackrel{\text { def }}{=}-a Q-b\left(Q^{*} \cdot Q+Q \cdot Q^{*}-\frac{2}{3}\left(Q^{*}: Q\right) \mathbf{I}\right)+c\left(\left|Q^{*}\right|^{2} Q+2\left(Q^{*}: Q\right) Q^{*}\right), \\
& \mathcal{P}_{3}(Q) \stackrel{\text { def }}{=}-b\left(Q^{2}+\frac{b}{3}|Q|^{2} \mathbf{I}\right)+c\left(|Q|^{2} Q+|Q|^{2} Q^{*}+2\left(Q: Q^{*}\right) Q\right) .
\end{aligned}
$$

Since for some constant vector $\mathbf{n}^{*} \in \mathbb{S}^{2}, Q^{*}=S\left(\mathbf{n}^{*} \mathbf{n}^{*}-\frac{1}{3} \mathbf{I}\right)$ is a critical point of $\mathcal{T}(Q)$, from Lemma 2.4 we get $\mathcal{T}\left(Q^{*}\right)=0$.

Multiplying the first equation in (3.1) by $Q_{\varepsilon}-Q^{*}$ and taking the $L^{2}$-inner product, we obtain

$$
\begin{aligned}
& \left\langle J \ddot{Q}_{\varepsilon}+\mu_{1} \dot{Q}_{\varepsilon}, Q_{\varepsilon}-Q^{*}\right\rangle+\left\langle\mathcal{L}\left(Q_{\varepsilon}\right), \mathcal{J}_{\varepsilon}\left(Q_{\varepsilon}-Q^{*}\right)\right\rangle \\
& =\underbrace{\left\langle-\frac{\mu_{2}}{2} \mathbf{D}_{\varepsilon}+\mu_{1}\left[\boldsymbol{\Omega}_{\varepsilon}, Q_{\varepsilon}\right], \mathcal{J}_{\varepsilon}\left(Q_{\varepsilon}-Q^{*}\right)\right\rangle}_{I_{1}} \underbrace{\left\langle\mathcal{T}\left(Q_{\varepsilon}\right), \mathcal{J}_{\varepsilon}\left(Q_{\varepsilon}-Q^{*}\right)\right\rangle}_{I_{2}} .
\end{aligned}
$$

Using the fact that $\langle[\Omega, Q], Q\rangle=0$, the estimate of $I_{1}$ can be calculated as

$$
\begin{aligned}
I_{1} & =\left\langle-\frac{\mu_{2}}{2} \mathbf{D}_{\varepsilon}+\mu_{1}\left[\boldsymbol{\Omega}_{\varepsilon}, Q^{*}\right], \mathcal{J}_{\varepsilon}\left(Q_{\varepsilon}-Q^{*}\right)\right\rangle \\
& \leq C\left\|\nabla \mathbf{v}_{\varepsilon}\right\|_{L^{2}}\left\|Q_{\varepsilon}-Q^{*}\right\|_{L^{2}} \leq C_{\delta} \mathcal{E}+\delta\left\|\nabla \mathbf{v}_{\varepsilon}\right\|_{L^{2}}^{2} .
\end{aligned}
$$


The term $I_{2}$ can be handled as

$$
\begin{aligned}
I_{2} & =-\left\langle\mathcal{T}\left(\widetilde{Q}_{\varepsilon}+Q^{*}\right), \mathcal{J}_{\varepsilon} \widetilde{Q}_{\varepsilon}\right\rangle=-\left\langle\mathcal{H}_{Q^{*}}\left(\widetilde{Q}_{\varepsilon}\right), \mathcal{J}_{\varepsilon} \widetilde{Q}_{\varepsilon}\right\rangle-\left\langle\mathcal{P}_{3}\left(\widetilde{Q}_{\varepsilon}\right), \mathcal{J}_{\varepsilon} \widetilde{Q}_{\varepsilon}\right\rangle \\
& \leq-\left\langle\mathcal{H}_{Q^{*}}\left(\widetilde{Q}_{\varepsilon}\right), \mathcal{J}_{\varepsilon} \widetilde{Q}_{\varepsilon}\right\rangle+C\left(\|\nabla Q\|_{L^{2}}+\|\nabla Q\|_{L^{2}}^{3}\right)\left\|\widetilde{Q}_{\varepsilon}\right\|_{L^{2}} \\
& \leq C\left(\mathcal{E}+\mathcal{E}^{2}\right) .
\end{aligned}
$$

Noticing that, for $Q \in \mathbb{S}_{0}^{3}$ and a constant tensor $Q^{*}$, we have

$$
\begin{aligned}
\left\langle\ddot{Q}, Q-Q^{*}\right\rangle & =\int_{\mathbb{R}^{3}}\left(\partial_{t}+v_{k} \partial_{k}\right) \dot{Q}_{i j}\left(Q_{i j}-Q_{i j}^{*}\right) \mathrm{d} \mathbf{x} \\
& =\int_{\mathbb{R}^{3}}\left(\partial_{t}\left(\dot{Q}_{i j}\left(Q_{i j}-Q_{i j}^{*}\right)\right)+v_{k} \partial_{k}\left(\dot{Q}_{i j}\left(Q_{i j}-Q_{i j}^{*}\right)\right)-\dot{Q}_{i j} \dot{Q}_{i j}\right) \mathrm{d} \mathbf{x} \\
& =\frac{1}{2} \frac{\mathrm{d}}{\mathrm{d} t}\left(\left\|\dot{Q}+Q-Q^{*}\right\|_{L^{2}}^{2}-\|\dot{Q}\|_{L^{2}}^{2}-\left\|Q-Q^{*}\right\|_{L^{2}}^{2}\right)-\|\dot{Q}\|_{L^{2}}^{2} .
\end{aligned}
$$

From (3.3) and (3.4) and the estimates of $I_{1}$ and $I_{2}$, we know that

$$
\begin{aligned}
\frac{1}{2} \frac{\mathrm{d}}{\mathrm{d} t}\left(J\left\|\dot{Q}_{\varepsilon}+Q_{\varepsilon}-Q^{*}\right\|_{L^{2}}^{2}-J\left\|\dot{Q}_{\varepsilon}\right\|_{L^{2}}^{2}+\left(\mu_{1}-J\right)\left\|Q_{\varepsilon}-Q^{*}\right\|_{L^{2}}^{2}\right) \\
\quad+L_{0}\left\|\nabla Q_{\varepsilon}\right\|_{L^{2}}^{2}+\left\langle\mathcal{H}_{Q^{*}}\left(Q_{\varepsilon}-Q^{*}\right), Q_{\varepsilon}-Q^{*}\right\rangle \\
\leq C\left(\mathcal{E}+\mathcal{E}^{2}\right)+\delta\left\|\nabla \mathbf{v}_{\varepsilon}\right\|_{L^{2}}^{2} .
\end{aligned}
$$

The basic energy dissipation in Lemma 2.3 tells us that

$$
\begin{aligned}
\frac{\mathrm{d}}{\mathrm{d} t} & \left(\int_{\mathbb{R}^{3}} \frac{1}{2}\left(\left|\mathbf{v}_{\varepsilon}\right|^{2}+J\left|\dot{Q}_{\varepsilon}\right|^{2}\right)+f_{b}\left(\widetilde{Q}_{\varepsilon}+Q^{*}\right)-f_{b}\left(Q^{*}\right)+f_{e}\left(\nabla Q_{\varepsilon}\right)\right) \mathrm{d} \mathbf{x} \\
= & -\beta_{1}\left\|Q_{\varepsilon}: \mathbf{D}_{\varepsilon}\right\|_{L^{2}}^{2}-\left(\beta_{4}-\frac{\mu_{2}^{2}}{4 \mu_{1}}-3 \delta\right)\left\|\mathbf{D}_{\varepsilon}\right\|_{L^{2}}^{2}-\left(\beta_{5}+\beta_{6}\right)\left\langle\mathbf{D}_{\varepsilon} \cdot Q_{\varepsilon}, \mathbf{D}_{\varepsilon}\right\rangle \\
& -2 \beta_{7}\left\|\mathbf{D}_{\varepsilon} \cdot Q_{\varepsilon}\right\|_{L^{2}}^{2}-\mu_{1}\left\|\dot{Q}_{\varepsilon}-\left[\boldsymbol{\Omega}_{\varepsilon}, Q_{\varepsilon}\right]+\frac{\mu_{2}}{2 \mu_{1}} \mathbf{D}_{\varepsilon}\right\|_{L^{2}}^{2}-\delta\left\|\nabla \mathbf{v}_{\varepsilon}\right\|_{L^{2}}^{2} \\
\leq & -\delta\left\|\nabla \mathbf{v}_{\varepsilon}\right\|_{L^{2}}^{2} .
\end{aligned}
$$

Thus, we multiply by 2 on (3.6) and then add it to (3.5), so that we obtain

$$
\begin{aligned}
\frac{\mathrm{d}}{\mathrm{d} t}( & \left\|\mathbf{v}_{\varepsilon}\right\|_{L^{2}}^{2}+\frac{J}{2}\left(\left\|\dot{Q}_{\varepsilon}+Q_{\varepsilon}-Q^{*}\right\|_{L^{2}}^{2}+\left\|\dot{Q}_{\varepsilon}\right\|_{L^{2}}^{2}\right) \\
& \left.+\frac{1}{2}\left(\mu_{1}-J\right)\left\|Q_{\varepsilon}-Q^{*}\right\|_{L^{2}}^{2}+2 \mathcal{L}(Q):\left(Q_{\varepsilon}-Q^{*}\right)\right) \\
\leq & -\delta\left\|\nabla \mathbf{v}_{\varepsilon}\right\|_{L^{2}}^{2}+C\left(\mathcal{E}+\mathcal{E}^{2}\right) .
\end{aligned}
$$

We now turn to the estimates of the higher order derivative for $\left(Q_{\varepsilon}, \mathbf{v}_{\varepsilon}\right)$. On the one hand, we take $\nabla^{s}$ on the first equation of (3.1) and multiply it by $\nabla^{s} \dot{Q}_{\varepsilon}$, integrate over $\mathbb{R}^{3}$ and by 
parts, then we arrive at

$$
\begin{aligned}
\frac{\mathrm{d}}{\mathrm{d} t}( & \left.\frac{J}{2}\left\|\nabla^{s} \dot{Q}_{\varepsilon}\right\|_{L^{2}}^{2}+\mathcal{L}\left(\nabla^{s} Q_{\varepsilon}\right): \nabla^{s} Q_{\varepsilon}\right)+\mu_{1}\left\|\nabla^{s} \dot{Q}_{\varepsilon}\right\|_{L^{2}}^{2} \\
= & -J\left\langle\nabla^{s}\left(\mathbf{v}_{\varepsilon} \cdot \nabla \dot{Q}_{\varepsilon}\right), \nabla^{s} \dot{Q}_{\varepsilon}\right\rangle-\left\langle\nabla^{s} \mathcal{T}\left(Q_{\varepsilon}\right), \nabla^{s} \dot{Q}_{\varepsilon}\right\rangle-\left\langle\nabla^{s} \mathcal{L}\left(Q_{\varepsilon}\right), \nabla^{s}\left(\mathbf{v}_{\varepsilon} \cdot \nabla Q_{\varepsilon}\right)\right\rangle \\
& \quad-\frac{\mu_{2}}{2}\left\langle\nabla^{s} \mathbf{D}_{\varepsilon}, \nabla^{s} \dot{Q}_{\varepsilon}\right\rangle+\mu_{1}\left\langle\nabla^{s}\left[\boldsymbol{\Omega}_{\varepsilon}, Q_{\varepsilon}\right], \nabla^{s} \dot{Q}_{\varepsilon}\right\rangle \\
& \stackrel{\text { def }}{=} \mathcal{I}_{1}+\mathcal{I}_{2}+\mathcal{I}_{3}+\mathcal{I}_{4}+\mathcal{I}_{5} .
\end{aligned}
$$

Using Lemma 2.2 and $\nabla \cdot \mathbf{v}_{\varepsilon}=0$, we obtain

$$
\begin{aligned}
\mathcal{I}_{1} & =-J\left\langle\nabla^{s}\left(\mathbf{v}_{\varepsilon} \cdot \nabla \dot{Q}_{\varepsilon}\right), \nabla^{s} \dot{Q}_{\varepsilon}\right\rangle+J\left\langle\mathbf{v}_{\varepsilon} \cdot \nabla \nabla^{s} \dot{Q}_{\varepsilon}, \nabla^{s} \dot{Q}_{\varepsilon}\right\rangle \\
& =-J\left\langle\llbracket \nabla^{s}, \mathbf{v}_{\varepsilon} \rrbracket \cdot \nabla \dot{Q}_{\varepsilon}, \nabla^{s} \dot{Q}_{\varepsilon}\right\rangle \\
& \leq J\left\|\llbracket \nabla^{s}, \mathbf{v}_{\varepsilon} \rrbracket \cdot \nabla \dot{Q}_{\varepsilon}\right\|_{L^{2}}\left\|\nabla^{s} \dot{Q}_{\varepsilon}\right\|_{L^{2}} \\
& \leq C\left\|\mathbf{v}_{\varepsilon}\right\|_{H^{s+1}}\left\|\dot{Q}_{\varepsilon}\right\|_{H^{s}}^{2} \leq C_{\delta}\left(\mathcal{E}^{\frac{3}{2}}+\mathcal{E}^{2}\right)+\delta\left\|\nabla \mathbf{v}_{\varepsilon}\right\|_{H^{s}}^{2} .
\end{aligned}
$$

From $\mathcal{T}\left(Q^{*}\right)=0$ and Lemma 2.1 , the term $\mathcal{I}_{2}$ can be derived,

$$
\begin{aligned}
\mathcal{I}_{2} & =-\left\langle\nabla^{s}\left(\mathcal{T}\left(Q_{\varepsilon}\right)-\mathcal{T}\left(Q^{*}\right)\right), \nabla^{s} \dot{Q}_{\varepsilon}\right\rangle \\
& =-\left\langle\mathcal{H}_{Q^{*}}\left(\nabla^{s} \widetilde{Q}_{\varepsilon}\right), \nabla^{s} \dot{Q}_{\varepsilon}\right\rangle-\left\langle\nabla^{s} \mathcal{P}_{3}\left(\widetilde{Q}_{\varepsilon}\right), \nabla^{s} \dot{Q}_{\varepsilon}\right\rangle \\
& \leq C\left(\left\|\nabla^{s} \widetilde{Q}_{\varepsilon}\right\|_{L^{2}}+\left\|\nabla^{s} \widetilde{Q}_{\varepsilon}\right\|_{L^{2}}^{2}+\left\|\nabla^{s} \widetilde{Q}_{\varepsilon}\right\|_{L^{2}}^{3}\right)\left\|\nabla^{s} \dot{Q}_{\varepsilon}\right\|_{L^{2}} \\
& \leq C\left(\mathcal{E}+\mathcal{E}^{\frac{3}{2}}+\mathcal{E}^{2}\right) .
\end{aligned}
$$

We observe that, for any $Q \in \mathbb{S}_{0}^{3}$,

$$
\begin{aligned}
-\langle\mathcal{L}(Q), \mathbf{v} \cdot \nabla Q\rangle & \\
= & \int_{\mathbb{R}^{3}} v_{j} Q_{k l, j}\left(L_{1} \Delta Q_{k l}+\frac{1}{2}\left(L_{2}+L_{3}\right)\left(Q_{k m, m l}+Q_{l m, m k}-\frac{2}{3} \delta_{k l} Q_{i j, i j}\right)\right) \mathrm{d} \mathbf{x} \\
= & \int_{\mathbb{R}^{3}}\left(-L_{1} v_{j} Q_{k l, m j} Q_{k l, m}-\frac{1}{2}\left(L_{2}+L_{3}\right)\left(v_{j} Q_{k l, l j} Q_{k m, m}+v_{j} Q_{k l, k j} Q_{l m, m}\right)\right. \\
& \left.-L_{1} v_{j, m} Q_{k l, j} Q_{k l, m}-\frac{1}{2}\left(L_{2}+L_{3}\right)\left(v_{j, l} Q_{k l, j} Q_{k m, m}+v_{j, k} Q_{k l, j} Q_{l m, m}\right)\right) \mathrm{d} \mathbf{x} \\
= & \int_{\mathbb{R}^{3}}\left(-L_{1} v_{j, m} Q_{k l, j} Q_{k l, m}-\frac{1}{2}\left(L_{2}+L_{3}\right)\left(v_{j, l} Q_{k l, j} Q_{k m, m}+v_{j, k} Q_{k l, j} Q_{l m, m}\right)\right) \mathrm{d} \mathbf{x} \\
\leq & C\|\nabla \mathbf{v}\|_{L^{\infty}}\|\nabla Q\|_{L^{2}}^{2} .
\end{aligned}
$$

By (3.9) and Lemma 2.1, the term $\mathcal{I}_{3}$ can be handled as follows:

$$
\begin{aligned}
\mathcal{I}_{3} & =-\left\langle\nabla^{s} \mathcal{L}\left(Q_{\varepsilon}\right), \mathbf{v}_{\varepsilon} \cdot \nabla \nabla^{s} Q_{\varepsilon}\right\rangle-\left\langle\nabla^{s} \mathcal{L}\left(Q_{\varepsilon}\right), \llbracket \nabla^{s}, \mathbf{v}_{\varepsilon} \rrbracket \cdot \nabla Q_{\varepsilon}\right\rangle \\
& \leq \mathcal{I}_{3}^{\prime}+C_{\delta}\left(\mathcal{E}^{2}+\mathcal{E}^{3}+\mathcal{E}^{4}\right)+\delta\left\|\nabla \mathbf{v}_{\varepsilon}\right\|_{H^{s}}^{2} .
\end{aligned}
$$


The term $\mathcal{I}_{5}$ can be calculated as

$$
\begin{aligned}
\mathcal{I}_{5} & =\mu_{1}\left\langle\nabla^{s}\left[\boldsymbol{\Omega}_{\varepsilon}, \widetilde{Q}_{\varepsilon}\right], \nabla^{s} \dot{Q}_{\varepsilon}\right\rangle+\mu_{1}\left\langle\left[\nabla^{s} \boldsymbol{\Omega}_{\varepsilon}, Q^{*}\right], \nabla^{s} \dot{Q}_{\varepsilon}\right\rangle \\
& \leq \mu_{1}\left\langle\left[\nabla^{s} \boldsymbol{\Omega}_{\varepsilon}, \widetilde{Q}_{\varepsilon}+Q^{*}\right], \nabla^{s} \dot{Q}_{\varepsilon}\right\rangle+C\left\|\widetilde{Q}_{\varepsilon}\right\|_{H^{s+1}}\left\|\mathbf{v}_{\varepsilon}\right\|_{H^{s}}\left\|\nabla^{s} \dot{Q}_{\varepsilon}\right\|_{L^{2}} \\
& \leq \mu_{1}\left\langle\left[\nabla^{s} \boldsymbol{\Omega}_{\varepsilon}, Q_{\varepsilon}\right], \nabla^{s} \dot{Q}_{\varepsilon}\right\rangle+C \mathcal{E}^{\frac{3}{2}} .
\end{aligned}
$$

On the other hand, we act the derivative operator $\nabla^{s}$ on the second equation of (3.1) and take $L^{2}$-inner product by multiplying $\nabla^{S} \mathbf{v}_{\varepsilon}$, then by integrating by parts we obtain

$$
\begin{aligned}
\frac{1}{2} \frac{\mathrm{d}}{\mathrm{d} t} & \left\|\nabla^{s} \mathbf{v}_{\varepsilon}\right\|_{L^{2}}^{2} \\
= & \left\langle\partial_{t} \nabla^{s} \mathbf{v}_{\varepsilon}, \nabla^{s} \mathbf{v}_{\varepsilon}\right\rangle \\
= & -\left\langle\nabla^{s}\left(\mathbf{v}_{\varepsilon} \cdot \nabla \mathbf{v}_{\varepsilon}\right), \nabla^{s} \mathbf{v}_{\varepsilon}\right\rangle-\left\langle\nabla ^ { s } \left(\beta_{1} Q_{\varepsilon}\left(Q_{\varepsilon}: \mathbf{D}_{\varepsilon}\right)+\beta_{4} \mathbf{D}_{\varepsilon}\right.\right. \\
& \left.\left.+\beta_{5} \mathbf{D}_{\varepsilon} \cdot Q_{\varepsilon}+\beta_{6} Q_{\varepsilon} \cdot \mathbf{D}_{\varepsilon}+\beta_{7}\left(\mathbf{D}_{\varepsilon} \cdot Q_{\varepsilon}^{2}+Q_{\varepsilon}^{2} \cdot \mathbf{D}_{\varepsilon}\right)\right), \nabla^{s+1} \mathbf{v}_{\varepsilon}\right\rangle \\
& -\frac{\mu_{2}}{2}\left\langle\nabla^{s}\left(\dot{Q}_{\varepsilon}-\left[\mathbf{\Omega}_{\varepsilon}, Q_{\varepsilon}\right]\right), \nabla^{s+1} \mathbf{v}_{\varepsilon}\right\rangle-\mu_{1}\left\langle\nabla^{s}\left[Q_{\varepsilon},\left(\dot{Q}_{\varepsilon}-\left[\mathbf{\Omega}_{\varepsilon}, Q_{\varepsilon}\right]\right)\right], \nabla^{s+1} \mathbf{v}_{\varepsilon}\right\rangle \\
& -\left\langle\nabla^{s} \sigma^{d}\left(Q_{\varepsilon}, Q_{\varepsilon}\right), \nabla^{s+1} \mathbf{v}_{\varepsilon}\right\rangle \\
& \stackrel{\text { def }}{=} \\
& \mathcal{J}_{1}+\mathcal{J}_{2}+\mathcal{J}_{3}+\mathcal{J}_{4}+\mathcal{J}_{5} .
\end{aligned}
$$

From Lemma 2.2, we can deduce that

$$
\begin{aligned}
\mathcal{J}_{1} & =\left\langle\llbracket \nabla^{s}, \mathbf{v}_{\varepsilon} \rrbracket \cdot \nabla \mathbf{v}_{\varepsilon}, \nabla^{s} \mathbf{v}_{\varepsilon}\right\rangle \leq C\left\|\mathbf{v}_{\varepsilon}\right\|_{H^{s+1}}\left\|\mathbf{v}_{\varepsilon}\right\|_{H^{s}}^{2} \\
& \leq C_{\delta}\left(\mathcal{E}^{\frac{3}{2}}+\mathcal{E}^{2}\right)+\delta\left\|\nabla \mathbf{v}_{\varepsilon}\right\|_{H^{s}}^{2} .
\end{aligned}
$$

The term $\mathcal{J}_{2}$ can be derived from Lemma 2.2,

$$
\begin{aligned}
\mathcal{J}_{2}= & -\left\langle\beta_{1} Q_{\varepsilon}\left(Q_{\varepsilon}: \nabla^{s} \mathbf{D}_{\varepsilon}\right)+\beta_{4} \nabla^{s} \mathbf{D}_{\varepsilon}+\beta_{5} \nabla^{s} \mathbf{D}_{\varepsilon} \cdot Q_{\varepsilon}+\beta_{6} Q_{\varepsilon} \cdot \nabla^{s} \mathbf{D}_{\varepsilon}\right. \\
& \left.+\beta_{7}\left(\nabla^{s} \mathbf{D}_{\varepsilon} \cdot Q_{\varepsilon}^{2}+Q_{\varepsilon}^{2} \cdot \nabla^{s} \mathbf{D}_{\varepsilon}\right), \nabla^{s} \mathbf{D}_{\varepsilon}\right\rangle-\beta_{1}\left\langle\llbracket \nabla^{s}, \widetilde{Q}_{\varepsilon} \widetilde{Q}_{\varepsilon}: \rrbracket \mathbf{D}_{\varepsilon}, \nabla^{s+1} \mathbf{v}_{\varepsilon}\right\rangle \\
& -\beta_{1}\left\langle\llbracket \nabla^{s}, Q^{*} \widetilde{Q}_{\varepsilon}: \rrbracket \mathbf{D}_{\varepsilon}, \nabla^{s+1} \mathbf{v}_{\varepsilon}\right\rangle-\beta_{1}\left\langle\llbracket \nabla^{s}, \widetilde{Q}_{\varepsilon} Q^{*}: \rrbracket \mathbf{D}_{\varepsilon}, \nabla^{s+1} \mathbf{v}_{\varepsilon}\right\rangle \\
& -\beta_{5}\left\langle\llbracket \nabla^{s}, \widetilde{Q}_{\varepsilon} \rrbracket \cdot \mathbf{D}_{\varepsilon}, \nabla^{s+1} \mathbf{v}_{\varepsilon}\right\rangle-\beta_{6}\left\langle\mathbf{D}_{\varepsilon} \cdot \llbracket \nabla^{s}, \widetilde{Q}_{\varepsilon} \rrbracket, \nabla^{s+1} \mathbf{v}_{\varepsilon}\right\rangle \\
& -\beta_{7}\left\langle\llbracket \nabla^{s}, \widetilde{Q}_{\varepsilon}^{2} \rrbracket \cdot \mathbf{D}_{\varepsilon}+\mathbf{D}_{\varepsilon} \cdot \llbracket \nabla^{s}, \widetilde{Q}_{\varepsilon}^{2} \rrbracket, \nabla^{s+1} \mathbf{v}_{\varepsilon}\right\rangle \\
& -\beta_{7}\left\langle\llbracket \nabla^{s}, \widetilde{Q}_{\varepsilon} \cdot Q^{*} \rrbracket \cdot \mathbf{D}_{\varepsilon}+\mathbf{D}_{\varepsilon} \cdot \llbracket \nabla^{s}, \widetilde{Q}_{\varepsilon} \cdot Q^{*} \rrbracket, \nabla^{s+1} \mathbf{v}_{\varepsilon}\right\rangle \\
& -\beta_{7}\left\langle\llbracket \nabla^{s}, Q^{*} \cdot \widetilde{Q}_{\varepsilon} \rrbracket \cdot \mathbf{D}_{\varepsilon}+\mathbf{D}_{\varepsilon} \cdot \llbracket \nabla^{s}, Q^{*} \cdot \widetilde{Q}_{\varepsilon} \rrbracket, \nabla^{s+1} \mathbf{v}_{\varepsilon}\right\rangle \\
\leq & -\beta_{1}\left\|Q_{\varepsilon}: \nabla^{s} \mathbf{D}_{\varepsilon}\right\|_{L^{2}}^{2}-\beta_{4}\left\|\nabla^{s} \mathbf{D}_{\varepsilon}\right\|_{L^{2}}^{2}-\left(\beta_{5}+\beta_{6}\right)\left\langle\nabla^{s} \mathbf{D}_{\varepsilon} \cdot Q_{\varepsilon}, \nabla^{s} \mathbf{D}_{\varepsilon}\right\rangle \\
& -2 \beta_{7}\left\|\nabla^{s} \mathbf{D}_{\varepsilon} \cdot Q_{\varepsilon}\right\|_{L^{2}}^{2}-\frac{\mu_{2}}{2}\left\langle\left[\nabla^{s} \mathbf{\Omega}_{\varepsilon}, Q_{\varepsilon}\right], \nabla^{s} \mathbf{D}_{\varepsilon}\right\rangle \\
& +C_{\delta}\left\|\mathbf{v}_{\varepsilon}\right\|_{H^{s}}^{2}\left(\left\|\widetilde{Q}_{\varepsilon}\right\|_{H^{s+1}}^{2}+\left\|\widetilde{Q}_{\varepsilon}\right\|_{H^{s+1}}^{4}\right)+\delta\left\|\nabla \mathbf{v}_{\varepsilon}\right\|_{H^{2}}^{2} .
\end{aligned}
$$


For $\mathcal{J}_{3}$, we get

$$
\begin{aligned}
\mathcal{J}_{3} & =-\frac{\mu_{2}}{2}\left\langle\nabla^{s} \dot{Q}_{\varepsilon}-\nabla^{s}\left[\boldsymbol{\Omega}_{\varepsilon}, \widetilde{Q}_{\varepsilon}\right], \nabla^{s+1} \mathbf{v}_{\varepsilon}\right\rangle-\frac{\mu_{2}}{2}\left\langle\nabla^{s} \dot{Q}_{\varepsilon}-\left[\nabla^{s} \boldsymbol{\Omega}_{\varepsilon}, Q^{*}\right], \nabla^{s+1} \mathbf{v}_{\varepsilon}\right\rangle \\
& \leq-\frac{\mu_{2}}{2}\left\langle\nabla^{s} \dot{Q}_{\varepsilon}-\left[\nabla^{s} \boldsymbol{\Omega}_{\varepsilon}, Q_{\varepsilon}\right], \nabla^{s} \mathbf{D}_{\varepsilon}\right\rangle+C_{\delta}\left\|\mathbf{v}_{\varepsilon}\right\|_{H^{s}}^{2}\left\|\widetilde{Q}_{\varepsilon}\right\|_{H^{s+1}}^{2}+\delta\left\|\nabla \mathbf{v}_{\varepsilon}\right\|_{H^{s}}^{2} .
\end{aligned}
$$

In the same way, the term $\mathcal{J}_{4}$ can be estimated,

$$
\begin{aligned}
\mathcal{J}_{4}= & -\mu_{1}\left\langle\nabla^{s}\left[\widetilde{Q}_{\varepsilon},\left(\dot{Q}_{\varepsilon}-\left[\boldsymbol{\Omega}_{\varepsilon}, \widetilde{Q}_{\varepsilon}\right]\right)\right], \nabla^{s+1} \mathbf{v}_{\varepsilon}\right\rangle-\mu_{1}\left\langle\nabla^{s}\left[\widetilde{Q}_{\varepsilon},\left(\dot{Q}_{\varepsilon}-\left[\boldsymbol{\Omega}_{\varepsilon}, Q^{*}\right]\right)\right], \nabla^{s+1} \mathbf{v}_{\varepsilon}\right\rangle \\
& -\mu_{1}\left\langle\left[Q^{*},\left(\nabla^{s} \dot{Q}_{\varepsilon}-\nabla^{s}\left[\boldsymbol{\Omega}_{\varepsilon}, \widetilde{Q}_{\varepsilon}\right]\right)\right], \nabla^{s+1} \mathbf{v}_{\varepsilon}\right\rangle-\mu_{1}\left\|\left[Q^{*}, \nabla^{s} \boldsymbol{\Omega}_{\varepsilon}\right]\right\|_{L^{2}}^{2} \\
\leq & -\mu_{1}\left\langle\left[\widetilde{Q}_{\varepsilon},\left(\nabla^{s} \dot{Q}_{\varepsilon}-\left[\nabla^{s} \boldsymbol{\Omega}_{\varepsilon}, \widetilde{Q}_{\varepsilon}\right]\right)\right], \nabla^{s+1} \mathbf{v}_{\varepsilon}\right\rangle-\mu_{1}\left\|\left[Q^{*}, \nabla^{s} \boldsymbol{\Omega}_{\varepsilon}\right]\right\|_{L^{2}}^{2} \\
& +C_{\delta}\left(\left\|\mathbf{v}_{\varepsilon}\right\|_{H^{s}}^{2}+\left\|\dot{Q}_{\varepsilon}\right\|_{H^{s}}^{2}+\left\|\mathbf{v}_{\varepsilon}\right\|_{H^{s}}^{2}\left\|\widetilde{Q}_{\varepsilon}\right\|_{H^{s+1}}^{2}\right)\left\|\widetilde{Q}_{\varepsilon}\right\|_{H^{s+1}}^{2}+\delta\left\|\nabla \mathbf{v}_{\varepsilon}\right\|_{H^{s}}^{2} \\
\leq & -\mu_{1}\left\langle\left[Q_{\varepsilon}, \nabla^{s} \boldsymbol{\Omega}_{\varepsilon}\right], \nabla^{s} \dot{Q}_{\varepsilon}-\left[\nabla^{s} \boldsymbol{\Omega}_{\varepsilon}, Q_{\varepsilon}\right]\right\rangle+C_{\delta}\left(\mathcal{E}^{2}+\mathcal{E}^{3}\right)+\delta\left\|\nabla \mathbf{v}_{\varepsilon}\right\|_{H^{s}}^{2} .
\end{aligned}
$$

Therefore, from (3.8) and (3.10), noting $\mathcal{I}_{3}^{\prime}+\mathcal{J}_{5}=0$ and gathering the previous estimates yields

$$
\begin{aligned}
\frac{\mathrm{d}}{\mathrm{d} t}( & \left.\frac{1}{2}\left\|\nabla^{s} \mathbf{v}_{\varepsilon}\right\|_{L^{2}}^{2}+\frac{J}{2}\left\|\nabla^{s} \dot{Q}_{\varepsilon}\right\|_{L^{2}}^{2}+\mathcal{L}\left(\nabla^{s} Q_{\varepsilon}\right): \nabla^{s} Q_{\varepsilon}\right) \\
\leq & -\mu_{1}\left\langle\nabla^{s} \dot{Q}_{\varepsilon}-\left[\nabla^{s} \boldsymbol{\Omega}_{\varepsilon}, Q_{\varepsilon}\right], \nabla^{s} \dot{Q}_{\varepsilon}\right\rangle-\frac{\mu_{2}}{2}\left\langle\nabla^{s} \mathbf{D}_{\varepsilon}, \nabla^{s} \dot{Q}_{\varepsilon}\right\rangle-\beta_{1}\left\|Q_{\varepsilon}: \nabla^{s} \mathbf{D}_{\varepsilon}\right\|_{L^{2}}^{2} \\
& -\beta_{4}\left\|\nabla^{s} \mathbf{D}_{\varepsilon}\right\|_{L^{2}}^{2}-\left(\beta_{5}+\beta_{6}\right)\left\langle\nabla^{s} \mathbf{D}_{\varepsilon} \cdot Q_{\varepsilon}, \nabla^{s} \mathbf{D}_{\varepsilon}\right\rangle-2 \beta_{7}\left\|\nabla^{s} \mathbf{D}_{\varepsilon} \cdot Q_{\varepsilon}\right\|_{L^{2}}^{2} \\
& -\frac{\mu_{2}}{2}\left\langle\left[\nabla^{s} \boldsymbol{\Omega}_{\varepsilon}, Q_{\varepsilon}\right], \nabla^{s} \mathbf{D}_{\varepsilon}\right\rangle-\frac{\mu_{2}}{2}\left\langle\nabla^{s} \dot{Q}_{\varepsilon}-\left[\nabla^{s} \boldsymbol{\Omega}_{\varepsilon}, Q_{\varepsilon}\right], \nabla^{s} \mathbf{D}_{\varepsilon}\right\rangle \\
& -\mu_{1}\left\langle\left[Q_{\varepsilon}, \nabla^{s} \boldsymbol{\Omega}_{\varepsilon}\right], \nabla^{s} \dot{Q}_{\varepsilon}-\left[\nabla^{s} \boldsymbol{\Omega}_{\varepsilon}, Q_{\varepsilon}\right]\right\rangle+C\left(\mathcal{E}^{3}+\mathcal{E}^{2}+\mathcal{E}^{3}\right)+6 \delta\left\|\nabla \mathbf{v}_{\varepsilon}\right\|_{H^{s}}^{2} \\
= & -\beta_{1}\left\|Q_{\varepsilon}: \nabla^{s} \mathbf{D}_{\varepsilon}\right\|_{L^{2}}^{2}-\left(\beta_{4}-\frac{\mu_{2}^{2}}{4 \mu_{1}}-7 \delta\right)\left\|\nabla^{s} \mathbf{D}_{\varepsilon}\right\|_{L^{2}}^{2}-\left(\beta_{5}+\beta_{6}\right)\left\langle\nabla^{s} \mathbf{D}_{\varepsilon} \cdot Q_{\varepsilon}, \nabla^{s} \mathbf{D}_{\varepsilon}\right\rangle \\
& -2 \beta_{7}\left\|\nabla^{s} \mathbf{D}_{\varepsilon} \cdot Q_{\varepsilon}\right\|_{L^{2}}^{2}-\mu_{1}\left\|\nabla^{s} \dot{Q}_{\varepsilon}-\left[\nabla^{s} \boldsymbol{\Omega}_{\varepsilon}, Q_{\varepsilon}\right]+\frac{\mu_{2}}{2 \mu_{1}} \nabla^{s} \mathbf{D}_{\varepsilon}\right\|_{L^{2}}^{2} \\
& -\delta\left\|\nabla \mathbf{v}_{\varepsilon}\right\|_{H^{s}}^{2}+C\left(\mathcal{E}+\mathcal{E}^{\frac{3}{2}}+\mathcal{E}^{2}+\mathcal{E}^{3}+\mathcal{E}^{4}\right) \\
\leq & -\delta\left\|\nabla \mathbf{v}_{\varepsilon}\right\|_{H^{s}}^{2}+C\left(\mathcal{E}+\mathcal{E}^{\frac{3}{2}}+\mathcal{E}^{2}+\mathcal{E}^{3}+\mathcal{E}^{4}\right) .
\end{aligned}
$$

Then, combining (3.7) and (3.11), we obtain

$$
\frac{\mathrm{d}}{\mathrm{d} t} \mathcal{E}(t)+\delta\left\|\nabla \mathbf{v}_{\varepsilon}\right\|_{H^{s}}^{2} \leq F(\mathcal{E}(t))
$$

where $F$ is an increasing function with $F(0)=0$, and is given by

$$
F(\mathcal{E}(t))=C\left(\mathcal{E}(t)+\mathcal{E}^{\frac{3}{2}}(t)+\mathcal{E}^{2}(t)+\mathcal{E}^{3}(t)+\mathcal{E}^{4}(t)\right) .
$$

Step 3. Existence of the solution. For $s \geq 2$, by virtue of (3.12), there exists $T>0$ depending only on $\mathcal{E}(0)$ such that, for any $t \in\left[0, \min \left(T, T_{\varepsilon}\right)\right]$,

$$
\mathcal{E}(t)+\delta\left\|\nabla \mathbf{v}_{\varepsilon}\right\|_{H^{s}}^{2} \leq 2 \mathcal{E}(0)
$$


where $\mathcal{E}(0)$ depends only on the initial data $\left(\mathbf{v}_{I}, Q_{I}\right)$. By a continuous argument we deduce that $T_{\varepsilon} \geq T$. Therefore, we get a uniform estimate for the approximate solution on $[0, T]$. Furthermore, the existence of the solution can be obtained by the standard compactness argument.

Step 4. Uniqueness of the solution. Assume that $\left(\mathbf{v}_{1}, Q_{1}\right)$ and $\left(\mathbf{v}_{2}, Q_{2}\right)$ are two strong solutions with the same initial data. We denote

$$
\begin{array}{ll}
\delta_{Q}=Q_{1}-Q_{2}, & \delta_{\dot{Q}}=\dot{Q}_{1}-\dot{Q}_{2}, \quad \delta_{\mathbf{v}}=\mathbf{v}_{1}-\mathbf{v}_{2}, \\
\delta_{\mathbf{D}}=\mathbf{D}_{1}-\mathbf{D}_{2}, & \delta_{\mathbf{\Omega}}=\boldsymbol{\Omega}_{1}-\boldsymbol{\Omega}_{2},
\end{array}
$$

where

$$
\delta_{\dot{Q}}=\partial_{t} \delta_{Q}+\mathbf{v}_{1} \cdot \nabla \delta_{Q}+\delta_{\mathbf{v}} \cdot \nabla Q_{2} .
$$

Taking the difference between the equations of the two solutions, we observe that $\left(\delta_{Q}, \delta_{\mathbf{v}}\right)$ satisfies the following system:

$$
\begin{aligned}
J\left(\partial_{t} \delta_{\dot{Q}}+\mathbf{v}_{1} \cdot \nabla \delta_{\dot{Q}}\right) & =-\mu_{1}\left(\delta_{\dot{Q}}-\left[\delta_{\boldsymbol{\Omega}}, Q_{2}\right]\right)-\mathcal{L}\left(\delta_{Q}\right)-\frac{\mu_{2}}{2} \delta_{\mathbf{D}}-J \delta_{\mathbf{v}} \cdot \nabla \dot{Q}_{2}+\delta_{\mathbf{F}_{1}} \\
\partial_{t} \delta_{\mathbf{v}}+\mathbf{v}_{1} \cdot \nabla \delta_{\mathbf{v}}= & -\nabla p+\nabla \cdot\left(\beta_{1} Q_{2}\left(Q_{2}: \delta_{\mathbf{D}}\right)+\beta_{4} \delta_{\mathbf{D}}+\beta_{5} \delta_{\mathbf{D}} \cdot Q_{2}\right. \\
& +\beta_{6} Q_{2} \cdot \delta_{\mathbf{D}}+\beta_{7}\left(\delta_{\mathbf{D}} \cdot Q_{2}^{2}+Q_{2}^{2} \cdot \delta_{\mathbf{D}}\right)+\frac{\mu_{2}}{2}\left(\delta_{\dot{Q}}-\left[\delta_{\mathbf{\Omega}}, Q_{2}\right]\right) \\
& \left.+\mu_{1}\left[Q_{2}, \delta_{\dot{Q}}-\left[\delta_{\boldsymbol{\Omega}}, Q_{2}\right]\right]\right)+\nabla \cdot \delta_{\mathbf{F}_{2}},
\end{aligned}
$$

where

$$
\begin{aligned}
\delta_{\mathbf{F}_{1}=} & \mu_{1}\left[\boldsymbol{\Omega}_{1}, \delta_{Q}\right]+a \delta_{Q}+b\left(Q_{1} \cdot \delta_{Q}+\delta_{Q} \cdot Q_{2}-\frac{1}{3}\left(Q_{1}: \delta_{Q}+\delta_{Q}: Q_{2}\right) \mathbf{I}\right) \\
& -c\left(\left|Q_{1}\right|^{2} \delta_{Q}+\left(Q_{1}: \delta_{Q}+\delta_{Q}: Q_{2}\right) Q_{2}\right), \\
\delta_{\mathbf{F}_{2}}= & \beta_{1}\left(\delta_{Q}\left(Q_{1}: \mathbf{D}_{1}\right)+Q_{2}\left(\delta_{Q}: \mathbf{D}_{1}\right)\right)+\beta_{5} \mathbf{D}_{1} \cdot \delta_{Q}+\beta_{6} \mathbf{D}_{1} \cdot \delta_{Q}+\frac{\mu_{2}}{2}\left[\boldsymbol{\Omega}_{1}, \delta_{Q}\right] \\
& +\mu_{1}\left[\delta_{Q}, \dot{Q}_{1}-\left[\boldsymbol{\Omega}_{1}, Q_{1}\right]\right]-\mu_{1}\left[Q_{2},\left[\boldsymbol{\Omega}_{1}, \delta_{Q}\right]\right]+\sigma^{d}\left(\delta_{Q}, Q_{i}\right)-\delta_{\mathbf{v}} \otimes \mathbf{v}_{2} .
\end{aligned}
$$

We denote $\widetilde{Q}_{i}=Q_{i}-Q^{*}$, then a direct calculation leads to the following estimates:

$$
\begin{aligned}
\left\|\delta_{\mathbf{F}_{1}}\right\|_{L^{2}} \leq & C\left(1+\left\|\nabla \mathbf{v}_{1}\right\|_{L^{3}}+\left\|\left(\widetilde{Q}_{1}, \widetilde{Q}_{2}\right)\right\|_{L^{\infty}}+\left\|\left(\widetilde{Q}_{1}, \widetilde{Q}_{2}\right)\right\|_{L^{\infty}}^{2}\right)\left(\left\|\delta_{Q}\right\|_{H^{1}}+\left\|\delta_{\mathbf{v}}\right\|_{L^{2}}\right), \\
\left\|\delta_{\mathbf{F}_{2}}\right\|_{L^{2}} \leq & C\left(\left\|\mathbf{v}_{2}\right\|_{L^{\infty}}+\left\|\nabla \mathbf{v}_{1}\right\|_{L^{3}}+\left\|\left(\widetilde{Q}_{1}, \widetilde{Q}_{2}\right)\right\|_{L^{\infty}}\left\|\nabla \mathbf{v}_{1}\right\|_{L^{3}}+\left\|\dot{Q}_{1}\right\|_{H^{1}}\right. \\
& \left.+\left\|\left(\nabla Q_{1}, \nabla Q_{2}\right)\right\|_{L^{\infty}}\right)\left(\left\|\delta_{Q}\right\|_{H^{1}}+\left\|\delta_{\mathbf{v}}\right\|_{L^{2}}\right) .
\end{aligned}
$$

For the system (3.13)-(3.14), making an $L^{2}$-energy estimate for $\left(\delta_{\dot{Q}}, \delta_{\mathbf{v}}\right)$, we obtain

$$
\begin{aligned}
\frac{1}{2} & \frac{\mathrm{d}}{\mathrm{d} t}\left(\left\|\delta_{\mathbf{v}}\right\|_{L^{2}}^{2}+J\left\|\delta_{\dot{Q}}\right\|_{L^{2}}^{2}+\left\langle\mathcal{L}\left(\delta_{Q}\right), \delta_{Q}\right\rangle\right) \\
\quad & =-\mu_{1}\left\langle\delta_{\dot{Q}}-\left[\delta_{\Omega}, Q_{2}\right], \delta_{\dot{Q}}\right\rangle-\left\langle\mathcal{L}\left(\delta_{Q}\right), \mathbf{v}_{1} \cdot \nabla \delta_{Q}+\delta_{\mathbf{v}} \cdot \nabla Q_{2}\right\rangle
\end{aligned}
$$




$$
\begin{aligned}
& -\frac{\mu_{2}}{2}\left\langle\delta_{\mathbf{D}}, \delta_{\dot{Q}}\right\rangle-J\left\langle\delta_{\mathbf{v}} \cdot \nabla \dot{Q}_{2}, \delta_{\dot{Q}}\right\rangle+\left\langle\delta_{\mathbf{F}_{1}}, \delta_{\dot{Q}}\right\rangle-\beta_{1}\left\|Q_{2}: \delta_{\mathbf{D}}\right\|_{L^{2}}^{2} \\
& -\beta_{4}\left\|\delta_{\mathbf{D}}\right\|_{L^{2}}^{2}-\left\langle\beta_{5} \delta_{\mathbf{D}} \cdot Q_{2}+\beta_{6} Q_{2} \cdot \delta_{\mathbf{D}}, \nabla \delta_{\mathbf{v}}\right\rangle-2 \beta_{7}\left\|\delta_{\mathbf{D}} \cdot Q_{2}^{2}\right\|_{L^{2}}^{2} \\
& -\frac{\mu_{2}}{2}\left\langle\delta_{\dot{Q}}-\left[\delta_{\boldsymbol{\Omega}}, Q_{2}\right], \nabla \delta_{\mathbf{v}}\right\rangle-\mu_{1}\left\langle\left[Q_{2}, \delta_{\dot{Q}}-\left[\delta_{\boldsymbol{\Omega}}, Q_{2}\right]\right], \nabla \delta_{\mathbf{v}}\right\rangle-\left\langle\delta_{\mathbf{F}_{2}}, \nabla \delta_{\mathbf{v}}\right\rangle \\
= & -\beta_{1}\left\|Q_{2}: \delta_{\mathbf{D}}\right\|_{L^{2}}^{2}-\left(\beta_{4}-\frac{\mu_{2}^{2}}{4 \mu_{1}}\right)\left\|\delta_{\mathbf{D}}\right\|_{L^{2}}^{2}-\left(\beta_{5}+\beta_{6}\right)\left\langle\delta_{\mathbf{D}} \cdot Q_{2}, \delta_{\mathbf{D}}\right\rangle \\
& -2 \beta_{7}\left\|\delta_{\mathbf{D}} \cdot Q_{2}\right\|_{L^{2}}^{2}-\mu_{1}\left\|\delta_{\dot{Q}}-\left[\delta_{\mathbf{\Omega}}, Q_{2}\right]+\frac{\mu_{2}}{2 \mu_{1}} \delta_{\mathbf{D}}\right\|_{L^{2}}^{2}+\left\langle\delta_{\mathbf{F}_{1}}, \delta_{\dot{Q}}\right\rangle \\
& -\left\langle\mathcal{L}\left(\delta_{Q}\right), \mathbf{v}_{1} \cdot \nabla \delta_{Q}+\delta_{\mathbf{v}} \cdot \nabla Q_{2}\right\rangle-J\left\langle\delta_{\mathbf{v}} \cdot \nabla \dot{Q}_{2}, \delta_{\dot{Q}}\right\rangle-\left\langle\delta_{\mathbf{F}_{2}}, \nabla \delta_{\mathbf{v}}\right\rangle .
\end{aligned}
$$

From (3.9) we get

$$
\begin{aligned}
& -\left\langle\mathcal{L}\left(\delta_{Q}\right), \mathbf{v}_{1} \cdot \nabla \delta_{Q}+\delta_{\mathbf{v}} \cdot \nabla Q_{2}\right\rangle \\
& \quad \leq C\left\|\nabla \mathbf{v}_{1}\right\|_{L^{\infty}}\left\|\nabla \delta_{Q}\right\|_{L^{2}}^{2}+C_{\delta}\left\|\nabla Q_{2}\right\|_{L^{\infty}}^{2}\left\|\nabla \delta_{Q}\right\|_{L^{2}}^{2}+\delta\left\|\nabla \delta_{\mathbf{v}}\right\|_{L^{2}}^{2} .
\end{aligned}
$$

Using the Sobolev embeddings $H^{1}\left(\mathbb{R}^{3}\right) \hookrightarrow L^{6}\left(\mathbb{R}^{3}\right)$ and $H^{1}\left(\mathbb{R}^{3}\right) \hookrightarrow L^{3}\left(\mathbb{R}^{3}\right)$, we find

$$
\begin{aligned}
-J\left\langle\delta_{\mathbf{v}} \cdot \nabla \dot{Q}_{2}, \delta_{\dot{Q}}\right\rangle & \leq C\left\|\delta_{\mathbf{v}}\right\|_{L^{3}}\left\|\nabla \dot{Q}_{2}\right\|_{L^{6}}\left\|\delta_{\dot{Q}}\right\|_{L^{2}} \leq C\left\|\delta_{\mathbf{v}}\right\|_{H^{1}}\left\|\nabla \dot{Q}_{2}\right\|_{H^{1}}\left\|\delta_{\dot{Q}}\right\|_{L^{2}} \\
& \leq C\left(1+\left\|\dot{Q}_{2}\right\|_{H^{2}}\right)\left(\left\|\delta_{\mathbf{v}}\right\|_{L^{2}}^{2}+\left\|\delta_{\dot{Q}}\right\|_{L^{2}}^{2}\right)+\delta\left\|\nabla \delta_{\mathbf{v}}\right\|_{L^{2}}^{2} .
\end{aligned}
$$

Consequently, from (3.15) and the above estimates and using the dissipation relation, for $i=1,2$, we have

$$
\begin{gathered}
\frac{1}{2} \frac{\mathrm{d}}{\mathrm{d} t}\left(\left\|\delta_{\mathbf{v}}\right\|_{L^{2}}^{2}+J\left\|\delta_{\dot{Q}}\right\|_{L^{2}}^{2}+\left\langle\mathcal{L}\left(\delta_{Q}\right), \delta_{Q}\right\rangle\right)+\delta\left\|\nabla \delta_{\mathbf{v}}\right\|_{L^{2}}^{2} \\
\quad \leq C\left(\mathbf{v}_{i}, \widetilde{Q}_{i}, \delta\right)\left(\left\|\delta_{\mathbf{v}}\right\|_{L^{2}}^{2}+\left\|\delta_{Q}\right\|_{H^{1}}^{2}+\left\|\delta_{\dot{Q}}\right\|_{L^{2}}^{2}\right) .
\end{gathered}
$$

In addition, multiplying Eq. (3.13) by $\delta_{Q}$ and taking the $L^{2}$-inner product, using integration by parts, then we have

$$
\begin{aligned}
& \frac{\mathrm{d}}{\mathrm{d} t}\left(J\left\langle\delta_{\dot{Q}}, \delta_{Q}\right\rangle+\frac{\mu_{1}}{2}\left\|\delta_{Q}\right\|_{L^{2}}^{2}\right)+\left\langle\mathcal{L}\left(\delta_{Q}\right), \delta_{Q}\right\rangle \\
&=J\left\|\delta_{\dot{Q}}\right\|_{L^{2}}^{2}-J\left\langle\delta_{\dot{Q}}, \delta_{\mathbf{v}} \cdot \nabla Q_{2}\right\rangle-\mu_{1}\left\langle\delta_{\mathbf{v}} \cdot \nabla Q_{2}-\left[\delta_{\Omega}, Q_{2}\right], \delta_{Q}\right\rangle \\
& \quad-J\left\langle\delta_{\mathbf{v}} \cdot \nabla \dot{Q}_{2}, \delta_{Q}\right\rangle-\frac{\mu_{2}}{2}\left\langle\delta_{\mathbf{D}}, \delta_{Q}\right\rangle+\left\langle\delta_{\mathbf{F}_{1}}, \delta_{Q}\right\rangle \\
& \leq C_{\delta}\left(1+\left\|\nabla Q_{2}\right\|_{L^{\infty}}^{2}+\left\|\widetilde{Q}_{2}\right\|_{L^{\infty}}+\left\|\dot{Q}_{2}\right\|_{H^{2}}^{2}\right)\left(\left\|\delta_{\mathbf{v}}\right\|_{L^{2}}^{2}+\left\|\delta_{\dot{Q}}\right\|_{L^{2}}^{2}+\left\|\delta_{Q}\right\|_{H^{1}}^{2}\right) \\
&+\frac{\delta}{2}\left\|\nabla \delta_{\mathbf{v}}\right\|_{L^{2}}^{2}+\left\|\delta_{\mathbf{F}_{1}}\right\|_{L^{2}}\left\|\delta_{Q}\right\|_{L^{2}} \\
& \leq C\left(\delta, \widetilde{Q}_{i}, \mathbf{v}_{i}\right)\left(\left\|\delta_{\mathbf{v}}\right\|_{L^{2}}^{2}+\left\|\delta_{\dot{Q}}\right\|_{L^{2}}^{2}+\left\|\delta_{Q}\right\|_{H^{1}}^{2}\right)+\frac{\delta}{2}\left\|\nabla \delta_{\mathbf{v}}\right\|_{L^{2}}^{2} .
\end{aligned}
$$


Hence, combining (3.16) and (3.17) leads to

$$
\begin{aligned}
\frac{1}{2} \frac{\mathrm{d}}{\mathrm{d} t}( & \left.\left\|\delta_{\mathbf{v}}\right\|_{L^{2}}^{2}+J\left\|\delta_{\dot{Q}}+\delta_{Q}\right\|_{L^{2}}^{2}+\left(\mu_{1}-J\right)\left\|\delta_{Q}\right\|_{L^{2}}^{2}+\left\langle\mathcal{L}\left(\delta_{Q}\right), \delta_{Q}\right\rangle\right) \\
& +\left\langle\mathcal{L}\left(\delta_{Q}\right), \delta_{Q}\right\rangle+\frac{\delta}{2}\left\|\nabla \delta_{\mathbf{v}}\right\|_{L^{2}}^{2} \\
\leq & C\left(\delta, \widetilde{Q}_{i}, \mathbf{v}_{i}\right)\left(\left\|\delta_{\mathbf{v}}\right\|_{L^{2}}^{2}+\left\|\delta_{\dot{Q}}\right\|_{L^{2}}^{2}+\left\|\delta_{Q}\right\|_{H^{1}}^{2}\right) .
\end{aligned}
$$

Since $J \ll \mu_{1}$, we obtain

$$
\begin{aligned}
& \frac{\mathrm{d}}{\mathrm{d} t}\left(\left\|\delta_{\mathbf{v}}\right\|_{L^{2}}^{2}+\left\|\delta_{\dot{Q}}\right\|_{L^{2}}^{2}+\frac{\mu_{1}}{2}\left\|\delta_{Q}\right\|_{L^{2}}^{2}+\left\langle\mathcal{L}\left(\delta_{Q}\right), \delta_{Q}\right\rangle\right) \\
& \leq C\left(\delta, \widetilde{Q}_{i}, \mathbf{v}_{i}\right)\left(\left\|\delta_{\mathbf{v}}\right\|_{L^{2}}^{2}+\left\|\delta_{\dot{Q}}\right\|_{L^{2}}^{2}+\left\|\delta_{Q}\right\|_{H^{1}}^{2}\right),
\end{aligned}
$$

thus, the Gronwall inequality implies that $\delta_{\mathbf{v}}(t)=0$ and $\delta_{Q}(t)=0$ on $[0, T]$.

Combining the above four steps, we complete the proof of Theorem 2.1.

\section{Conclusions}

In this paper, we are mainly concerned with the inertial Qian-Sheng Q-tensor model describing the nematic liquid crystal flow. The inertial term $J$ is responsible for the hyperbolic feature of the equation describing molecular orientation. Under the assumption of the initial data near uniaxial equilibrium, we investigate the existence and uniqueness of local in time strong solutions to the system. However, the global in time existence around the uniaxial equilibrium is rather difficult because the energy of the system is not strong enough to estimate the $L^{2}$-norm of the difference $Q-Q^{*}$. This will be left for our future work.

Acknowledgements

The authors would like to thank the anonymous referees for their valuable comments and suggestions to improve the quality of this paper.

\section{Funding}

Sirui Li is supported by the NSF of China under Grant No. 12061019 and No. 11601099, and by the Science and Technology Foundation of Guizhou Province of China under Grant No. [2017]1032, and by the Growth Foundation for Youth Science and Technology Talent of Educational Commission of Guizhou Province of China under Grant No. [2021]087.

\section{Availability of data and materials}

Not applicable.

Competing interests

The authors declare that they have no competing interests.

\section{Authors' contributions}

XW, SL and TW participated in the theoretical research and drafted the manuscript. All authors read and approved the final manuscript.

\section{Publisher's Note}

Springer Nature remains neutral with regard to jurisdictional claims in published maps and institutional affiliations.

Received: 28 September 2020 Accepted: 8 February 2021 Published online: 23 February 2021

\section{References}

1. Abels, H., Dolzmann, G., Liu, Y.: Well-posedness of a fully-coupled Navier-Stokes/Q-tensor system with inhomogeneous boundary data. SIAM J. Math. Anal. 46, 3050-3077 (2014) 
2. Abels, H., Dolzmann, G., Liu, Y.: Strong solutions for the Beris-Edwards model for nematic liquid crystals with homogeneous Dirichlet boundary conditions. Adv. Differ. Equ. 21, 109-152 (2016)

3. Beris, A.N., Edwards, B.J.: Thermodynamics of Flowing Systems with Internal Microstructure. Oxford Engrg. Sci. Ser, vol. 36. Oxford University Press, Oxford (1994)

4. De Anna, F., Zarnescu, A.: Global well-posedness and twist-wave solutions for the inertial Qian-Sheng model of liquid crystals. J. Differ. Equ. 264, 1080-1118 (2018)

5. De Gennes, P.G.: The Physics of Liquid Crystals. Clarendon Press, Oxford (1974)

6. Feireisl, E., Rocca, E., Schimperna, G., Zarnescu, A.: On a hyperbolic system arising in liquid crystals modeling. J. Hyperbolic Differ. Equ. 15, 15-35 (2018)

7. Han, J., Luo, Y., Wang, W., Zhang, P., Zhang, Z.: From microscopic theory to macroscopic theory: systematic study on modeling for liquid crystals. Arch. Ration. Mech. Anal. 215, 741-809 (2015)

8. Huang, J., Ding, S.: Global well-posedness for the dynamical Q-tensor model of liquid crystals. Sci. China Math. 58 1349-1366 (2015)

9. Li, S., Wang, W.: Rigorous justification of the uniaxial limit from the Qian-Sheng inertial Q-tensor theory to the Ericksen-Leslie theory. SIAM J. Math. Anal. 52, 4421-4468 (2020)

10. Li, S.-R., Wang, W., Zhang, P.: Local well-posedness and small Deborah limit of a molecule-based Q-tensor system. Discrete Contin. Dyn. Syst., Ser. B 20, 2611-2655 (2015)

11. Liu, Y., Wang, W.: On the initial boundary value problem of a Navier-Stokes/Q-tensor model for liquid crystals. Discrete Contin. Dyn. Syst., Ser. B 23, 3879-3899 (2018)

12. Majumdar, A.: Equilibrium order parameters of nematic liquid crystals in the Landau-de Gennes theory. Eur. J. Appl. Math. 21, 181-203 (2010)

13. Mottram, N.J., Newton, C.: Introduction to Q-tensor theory (2014) arXiv:1409.3542

14. Paicu, M., Zarnescu, A.: Global existence and regularity for the full coupled Navier-Stokes and Q-tensor system. SIAM J. Math. Anal. 43, 2009-2049 (2011)

15. Paicu, M., Zarnescu, A.: Energy dissipation and regularity for a coupled Navier-Stokes and Q-tensor system. Arch. Ration. Mech. Anal. 203, 45-67 (2012)

16. Qian, T., Sheng, P.: Generalized hydrodynamic equations for nematic liquid crystals. Phys. Rev. E 58, 7475-7485 (1998)

17. Triebel, H.: Theory of Function Spaces. Monographs in Mathematics. Birkhäuser, Basel (1983)

18. Wang, W., Zhang, P., Zhang, Z.: Well-posedness of the Ericksen-Leslie system. Arch. Ration. Mech. Anal. 206, 953-995 (2012)

19. Wang, W., Zhang, P., Zhang, Z.: Rigorous derivation from Landau-de Gennes theory to Ericksen-Leslie theory. SIAM J. Math. Anal. 47, 127-158 (2015)

\section{Submit your manuscript to a SpringerOpen ${ }^{\circ}$ journal and benefit from:}

- Convenient online submission

- Rigorous peer review

- Open access: articles freely available online

- High visibility within the field

- Retaining the copyright to your article

Submit your next manuscript at $\gg$ springeropen.com 